Objectives: To evaluate the survival rate of DNS, adverse events and reasons for the DNS discontinuation.

Methods: This was a prospective observation study in patients with OP which ini-

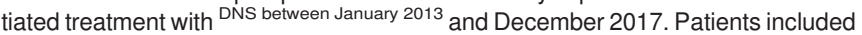
were followed up in the Rheumatology Nurse Clinic every six month. Demographics date, disease features, concomitant disease and treatments, adverse events and reasons for discontinuation were collected.

Results: We included 220 patients ( $80.5 \%$ women) with a mean age (range, SD) of $67.19(30-89,11.2)$ years. In average (range, SD), patients received 3.85 (1$11,2.2$ ) doses of DNS, with a mean duration of treatment (range, SD) of 23.03 (6$66,13.3)$ months. $191(86.8 \%)$ patients received also treatment with calcium and vitamin D supplements. Before the start of the treatment with DNS, 123 (55.9\%) patients had received another specific treatment for OP with mean previous treatment duration of 51.6 months. Previous fragility fractures were reported in 150 $(68.1 \%)$ patients, of whom 91 (41.3\%) patients had two or more fractures before starting treatment with DNS

Of all included the patients, 108 (49\%) patients had an inflammatory autoimmune disease (IAD) diagnosed. In addition, 100 (45.4\%) patients had concomitant biological and/or synthetic treatment and 77 (35\%) patients received concomitant treatment with corticosteroids.

During the treatment with DNS, 30 (13.6\%) patients had new fractures, $5(2.3 \%)$ patients had 2 fractures. Eleven fractures were vertebral, 3 of femur, one of radius and 21 other locations. There were no differences between patients with or without glucocorticoid treatment $(0.234)$.

The most frequent adverse events (AE) were infections in $88(40 \%)$ patients, muscle pain in $15(0.6 \%)$ patients, fatigue in $7(0.31 \%)$ patients, itching, heat and fever in $2(0.9 \%)$ patients and osteonecrosis of the jaw in $2(0.9 \%)$ patients. The 2 patients with osteonecrosis of the jaw had previous treatment with bisphosphonates for more than 24 months.

At 60 months, $185(84.1 \%)$ patients continued with DNS. In 37 (1.6\%) patients, DNS was discontinued; in 4 patents DNS was restarted. The reasons for suspension were hypercalciuria $1(0.04 \%)$, hypocalcemia $1(0.04 \%)$, local hypersensitivity reactions $4(0.18 \%)$, normalisation of BMD $5(0.2 \%)$, dental problems 11 $(0.4 \%)$ and others $17(0.7 \%)$. The mean $(\mathrm{SD}, 95 \% \mathrm{Cl})$ of DNS survival was 51.2 $(1.9 ; 47.3-55.1)$ months. There are no differences in the survival rates of DNS between patients with and without concomitant biologic therapy $(p=0.995)$.

Conclusions: The majority of patients who started treatment with DNS continue the treatment with good tolerance. The most frequent adverse effects were infections but they have not led to suspension of treatment.

Disclosure of Interest: None declared

DOI: 10.1136/annrheumdis-2018-eular.5531

\section{AB0986 MANAGEMENT OF OSTEOPOROSIS AFTER MAJOR FRACTURE IN A COHORT OF WOMEN AGED OVER 50 IN A REAL LIFE SETTING}

A. Nutz ${ }^{1}$, S. Bastide ${ }^{2,3}$, F. Flaisler ${ }^{1}$, C. Gaujoux-Viala ${ }^{1,2} .{ }^{1}$ Rheumatology, Nîmes University Hospital, NIMES; ${ }^{2}$ EA2415, Montpellier University, MONTPELLIER; ${ }^{3}$ Biostatics, Epidemiology, Public Health and Innovation in Methodology (BESPIM), Nîmes University Hospital, NIMES, France

Background: Osteoporosis is the most common metabolic bone disease. Fractures constitute a major health concern because prior fractures are associated with an increased risk of subsequent fracture. Moreover, osteoporotic fractures have been associated with increased mortality risk. ${ }^{1}$ Hence secondary fracture prevention is recommended. Anti-osteoporotic drugs are available, but in the last decade an alarming decrease of anti-osteoporotic therapy use was observed. ${ }^{2}$ Objectives: The objective of this study was to analyse the management of osteoporosis after major fracture (hip, vertebral and humerus fractures) in women aged over 50 in a real life setting.

Methods: We conducted an observational study on all women over 50 years of age who experienced a major fracture at Nîmes University Hospital in 2015 identified by informatic codes. Only fragility fractures were included. Data collected prospectively in the computerised medical files were extracted: prescription of a postmenopausal osteoporosis therapy; therapy initiation; prescription and completion of generalised bone mineral density testing; and prescription and completion of blood sample tests to look for secondary osteoporosis. We also recorded the department in which the hospitalizations occurred and the fracture localization. Results: Of 375 women over 50 with a registered fracture in 2015, 264 were included. The exclusion criteria were women with traumatic fracture or minor osteoporotic fracture. The median age was 84 years old. The most common major fractures were hip (52.3\%), humerus $(20.8 \%)$ and spine $(18.9 \%)$.

Forty-five (17.0\%) patients had experienced a fracture prior to the 2015 fracture. Only 12 patients (4.5\%: IC95\%[2.4-7.8]) had an anti-osteoporotic drug prescription at the end of the hospitalisation and only 45 women (17\%) had anti-osteoporotic drug prescription planned later. The median period until anticipated prescription was 9 days and the median period until prescription in practice was 18 days.

Assessment of bone mineral density was planned later in only 23 patients $(8.7 \%$ : IC95\% [5.6-12.8]). Blood sample tests were realised in 49 women (18.6\%: IC $95 \%$ [14.1-23.8]).

In surgical department, anti-osteoporotic drug prescription was planned in only 3 women (1.5\%) compared with 40 women $(56.3 \%)$ in medical departments.

Conclusions: A large majority of women with osteoporotic major fracture are not receiving appropriate therapy and recommended management in 2015 in Nîmes University Hospital. The rate is dependent on the department in which patients are hospitalised.

The results of our study highlight the urgent need for optimisation of osteoporotic fracture management, especially in the surgical department.

\section{REFERENCES:}

[1] Katsoulis M, Benetou V, Karapetyan T, Feskanich D, Grodstein F, Petters son-Kymmer $U$, et al. Excess mortality after hip fracture in elderly persons from Europe and the USA: the CHANCES project. J Intern Med. 2017 Mar;281(3):300-10.

[2] Van der Velde RY, Wyers CE, Teesselink E, Geusens PPMM, van den Bergh JPW, de Vries F, et al. Trends in oral anti-osteoporosis drug prescription in the United Kingdom between 1990 and 2012: Variation by age, sex, geographic location and ethnicity. Bone. 2017:94:50-5.

Disclosure of Interest: None declared

DOI: 10.1136/annrheumdis-2018-eular.5761

\section{AB0987 FREQUENCY OF UTILISATION OF THE CENTRAL DXA BONE DENSITOMETRY IN PATIENTS WITH MULTIPLE SCLEROSIS}

A.N. Klimo. Specialized hospital of rehabilitation "Banja Kanjiza", Kanjiza, Serbia

Background: Multiple sclerosis patients can have a higher risk from occurrence of osteoporosis. Reduced bone mass density can be related to a cumulative effect of different factors, most common ones being physical inactivity, reduced intake of vitamin $\mathrm{D}$ and use of medications such as glucocorticoids.

Objectives: The aim of this research was to explore the level of awareness in patients and physicians on the significance of the utilisation of DXA bone densitometry in patients with multiple sclerosis.

Methods: The observational analytical cross-section study included 366 multiple sclerosis patients on stationary treatment at the Special rehabilitation hospita "Banja Kanjiža" in Kanjiža in the period between 2013 and 2017. The following parameters were observed in patients: sex, age, duration and form of basic disease, the level on the Kurtzke Expanded Disability Status Scale, utilisation of glucocorticoids, occurrence of pathological fractures and intake of vitamin D, i.e. of medication for the treatment of osteoporosis in order to determine their impact on the frequency of the low bone mineral density (BMD). Statistical data processing and analysis was conducted in the SPSS ver 20.0 program by IBM corporation.

Results: In the period in question, an average of 128 multiple sclerosis patients were treated, out of those $62.3 \%(n=228)$ with relapsing-remitting type of disease and $n=366$ first time patients. Within the given period, $36 \%$ more women than men were rehabilitated ( $f=249$ vs. $m=117$ ). During the five-year long period of observation of said patients, only $8.5 \%(n=31)$ of patients with different levels of bone metabolic disorders established underwent central DXA bone densitometry. Pathological fracture on a small trauma was suffered in $6.8 \%(n=25)$ patients. Of the abovementioned parameters, only the female sex $\left(X^{2}=84.492 ; p<0.001\right)$ and age $(t=2.100 ; p=0.036)$ statistically significantly influenced the occurrence of low bone mineral density.

Conclusions: It is necessary to increase the level of health education of multiple sclerosis patients on the consequences of low bone mineral density. The highest risk of osteoporotic fracture is in older women suffering from multiple sclerosis.

Disclosure of Interest: None declared

DOI: 10.1136/annrheumdis-2018-eular.1632 